\title{
Evaluation of the Fine Structure Constant
}

\author{
Adriano Alippi \\ Dept. of Basic and Applied Sciences for Engineering, Sapienza University of Rome, Rome, Italy \\ Email: adriano.alippi@uniroma1.it
}

How to cite this paper: Alippi, A. (2020) Evaluation of the Fine Structure Constant. Journal of Modern Physics, 11, 1918-1925. https://doi.org/10.4236/jmp.2020.1112120

Received: November 5, 2020

Accepted: December 4, 2020

Published: December 7, 2020

Copyright (c) 2020 by author(s) and Scientific Research Publishing Inc. This work is licensed under the Creative Commons Attribution International License (CC BY 4.0).

http://creativecommons.org/licenses/by/4.0/

\begin{abstract}
An equation is given for analytically defining the value of the fine structure constant, whose derivation follows two main steps, relative to the generation of electric charges and to the polarizability of vacuum due to virtual dipoles. The obtained value matches the experimental one by a factor lower than the relative standard uncertainty produced by the National Institute of Standards and Technology (NIST).
\end{abstract}

\section{Keywords}

Fine Structure Constant, Virtual Particles, Vacuum Permittivity

\section{Introduction}

The search for a theory that might give reasons for the value of constants in natural phenomena dates from the very beginning of man observing nature processes, and as new physics was going to be introduced into human knowledge, the problem stepped forward to include more fundamental constants. The modern problem was lucidly set by P. A. M. Dirac in a paper dated 1937 [1], where proper mention was given of the frantic efforts made by A. Eddington [2] in the field. Today, the values of fundamental constants are known with ever increasing precision [3] and efforts to give reasons of any single quantity or any link among different quantities are constantly under way and it would be inevitably tedious to cite even a limited group of them.

Probably, the so-called fine structure constant, from the name Arnold Sommerfeld used for it in 1916 in extending the Bohr model of the atom [4], stands as the most tricky character of the drama, for its receding way to be tracked and its adimensional nature. An endless number of articles treat the history and mystery of the constant and here we do only refer to a couple of them, relative to the earliest and to the most recent times [5] [6].

The present paper reports the process aimed at evaluating the fine structure 
constant with a value that matches the experimental one reported by the National Institute of Standards and Technology (NIST) by a relative factor $8.4 \times 10^{-11}$, which is lower than the relative standard uncertainty [3]. That stands as a strong support for publicizing the obtained equation, in view of proper comments on the underlying procedure.

The question naively rises as to whether an Occam's razor approach would be successful in the struggle for defining the constant value. The way reported here follows two main steps, derived by the assumption that virtual particles [7] modify the electric permittivity of the vacuum, therefore the implicit value of the constant, as it results from the measured values of the contributing physical magnitudes. The two steps refer to the wave function describing the generation of electric charges in the so-called pair production phenomenon, and to the reduced permittivity of the vacuum in the absence of virtual particles. The procedure is based on the fundamental laws of electromagnetism and basic quantum physics and, as such, the referred literature is limited to basic texts.

\section{Evaluating the Charge Value}

The generation of an electric charge $q$ is only possible with the contemporary generation of a second charge $-q$, allocated in the same point or, more properly, whose space-time distribution of the wave function completely matches that of the first charge, apart from a phase factor, that would be only observable at the interfering of the two waves. The two charges thus created do not produce any electric field in space, whose time variation would have a spherical symmetry that would not be consistent with the Maxwell laws of electromagnetism. However, they successively separate and create a dipole field in space. The charge value that attains the maximum probability is computed and successively that value is selected which allows the particles to separate. To this aim, let us construct the function $\psi$ of either charge, say the positive one $q$, by applying the operator $\hat{q}$, corresponding to the derivative of $\psi$ with respect to the conjugate variable of the charge itself, written as $\Theta$ :

$$
-i \hbar \frac{\partial \psi}{\partial \Theta}=q \psi(q, \Theta) .
$$

Solution of the equation is

$$
\psi=C \mathrm{e}^{i \frac{q \Theta}{\hbar}},
$$

with the constant $C$ to be determined by initial conditions. Function $\Theta$ should be such that its product with quantity $q$ shares the dimensions of the Planck constant $h$, equal then to those of the ratio between the electric flux $\Phi(E)$ and the particle velocity $V$. In addition, when generated, each charge would move away with velocity $\boldsymbol{v}$ in a direction opposite to that of the second charge and the electric field generated by it starts growing spherically outwards with velocity $c$ from the point of generation.

The flux of the electric field may then be calculated on a plane normal to the 
velocity, through the generation point. The surface for the evaluation of the flux can be closed with any half sphere surface at a proper distance where the electric field perturbation has not yet reached.

The flux derivative $\partial \Phi(\boldsymbol{E}) / \partial v$ depends neither on $v$ nor on time $t$, and stands for the $\Theta$ function to be considered, since the wave function in this way would not depend on space or time variables. Indeed, for a charge created at the origin of the coordinates at the time $t<0$, that moves away along the positive $x$ axis with a velocity $v$, the electric field in a point $\mathrm{P}=(0,0, z)$ of the $z$ axis at the time $t=0$, is [5]

$$
\boldsymbol{E}=-\frac{q}{4 \pi \varepsilon_{0}} \frac{\gamma}{r^{3}\left[1+\left(\gamma v_{r} / c\right)^{2}\right]^{3 / 2}} \boldsymbol{r}
$$

where $\boldsymbol{r}$ is the distance at the time $t=0$ between the charge and the point $\mathrm{P}, V_{r}$ the component of the velocity vector $\boldsymbol{V}$ along $\boldsymbol{r}, \boldsymbol{c}$ the velocity of light, $\varepsilon_{0}$ the dielectric constant and $\gamma$ the factor $\gamma=1 / \sqrt{1-\beta^{2}}$, with $\beta=v / c$. The electric field flux through the plane $x=0$ at time $t=0$ may be, then, calculated by considering the cylinder geometry of the problem to obtain

$$
\Phi(x=0, t=0)=\frac{q}{2 \varepsilon_{0}}(\beta-1) .
$$

The function $\Theta$ can now be computed as the derivative of the flux expression reported above, and that however shall be considered as a density of the function with respect to the solid angle $\Omega$, since the velocity $v$ can be oriented along any direction:

$$
\frac{\mathrm{d} \Theta}{\mathrm{d} \Omega}=\frac{\mathrm{d} \Phi}{\mathrm{d} v}
$$

In addition, a factor 2 has to be taken into consideration for the electric field flux and its derivative, since an equal additional amount to it is contributed by the second charge, entangled with the first one. Finally, one obtains

$$
\Theta=\frac{4 \pi q}{\varepsilon_{0} c} .
$$

that has to be inserted into Equation (2) to give

$$
\psi_{+}=C e^{4 \pi i \frac{q^{2}}{\varepsilon_{0} c h}}
$$

being $\psi_{+}$specified for the positive charge $q$. The wave function of the second charge of opposite sign $-q$ is equal to that of the first one, save for the sign in the exponent and a different constant $C$. With regard to these constants, the probability of the generation of both particles $P_{\text {pair }}=\left|\psi_{+}+\psi_{-}\right|^{2}$ vanishes at $q=0$, so that we may assume $C^{\prime}=-C$, and one may write

$$
P_{\text {pair }}=\left|\psi_{+}+\psi_{-}\right|^{2}=\left[2 \sin \left(\frac{4 \pi q}{q_{\mathrm{p}}}\right)^{2}\right]^{2},
$$

where the expression of the Planck charge unit $q_{\mathrm{P}}=\sqrt{2 \varepsilon_{0} c h}$ has been used. 
Such a probability value, however, which is a maximum at

$$
q_{\max }=\frac{q_{P}}{4 \sqrt{2 \pi}}=\frac{e}{4 \sqrt{2 \pi \alpha}}=1.1675 e,
$$

with $\alpha=\left(e / q_{\mathrm{P}}\right)^{2}$ the fine structure constant, is not relative to a pair of particles experimentally observable, but only to the generation of a pair of opposite charges departing one from the other. These are just virtual particles which, in order to be detectable, must separate one from the other, in a process that is counteracted by the electrostatic attractive force between them. The process has no interpretation in classical physics, but could be only described by quantum physics. The quantum potential of Bohmian theory [8], for instance, would allow to localize two pointlike particles in different places at the very same time, permitting a finite force to overtake the attractive effect. The separation of the particles makes the wave function to collapse into a definite state, which corresponds to the maximum of probability with a minimum work. Since the electrostatic attractive force depends on a factor $q^{2} / \varepsilon_{0}$, the probability function in Equation (8) could be multiplied by this inverse factor and the maximum of the obtained overall function defines the state of the free particles:

$$
P=P_{\text {gen }} \cdot P_{\text {sep }} \propto \frac{\sin ^{2}\left(\frac{4 \pi q}{q_{\mathrm{P}}}\right)^{2}}{\left(\frac{4 \pi q}{q_{\mathrm{P}}}\right)^{2}}=\left(\frac{\sin \xi^{2}}{\xi}\right)^{2}
$$

where $P_{\text {gen }}=P_{\text {pair }}$ is the probability of generating the pair and a similar notation $P_{\text {sep }}$ is used for the separation process, although this is not a probability function. It is worth pointing that the zeroes and maxima of the $P$ function do not depend on the multiplying coefficient of the single component functions, and this makes it possible to define the $P$ function in just one variable $\xi=4 \pi q / q_{\mathrm{p}}$, as reported in Equation (10).

The two functions $P_{\text {gen }}$ and $P_{\text {sep }}$ are represented in Figure 1 vs. the parameter

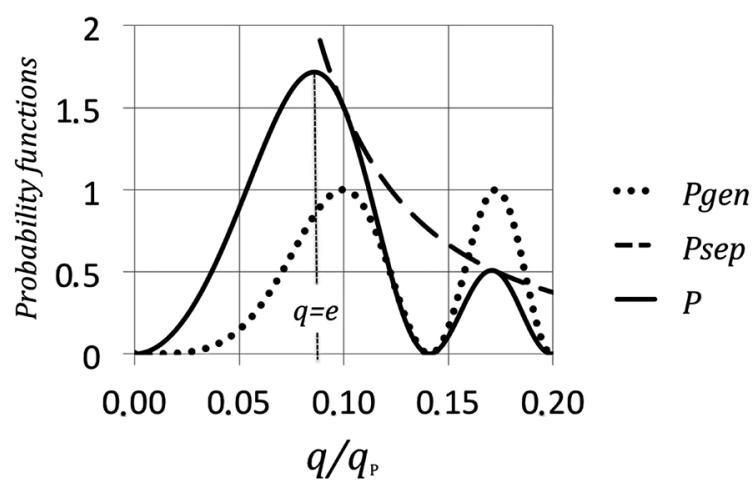

Figure 1. Probability functions vs. value of the charge, in Planck unit $q_{\mathrm{P}}=\sqrt{2 \varepsilon_{0} c h}$. Units for each component function are arbitrarily chosen, in as much as the positions of maxima and minima in the product of functions $P_{\text {gen }}$ and $P_{\text {sep }}$ are not sensitive to normalization factors. 
$q / q_{\mathrm{P}}=\xi /(4 \pi)$, where the two effects are identified, that counteract each other at low values of $\xi$, up to the first maximum, that is attained at $\xi_{\max }=$ 1.07961158997447. This value is not compatible with the known values of the involved constant and that is supposedly due to the presence of virtual particles in the vacuum, which affect the interaction between the true charges, by changing the electric permittivity of vacuum to its commonly known value $\varepsilon_{0}$.

Indeed, the maximum condition on function $P$ sets a constrain on all the electromagnetic quantities involved, $q, c$ and $\varepsilon_{0}$. The velocity $c$, however, is not to be considered affected by virtual particles, as it is suggested by its equality for electromagnetic and gravitational waves [9].

The $\xi_{\max }$ condition reduces then to a constrain on the electromagnetic interaction parameter $q^{2} / \varepsilon_{0}$. Charges originate with $\pm e$ values in an absolute vacuum that possesses an electric permittivity $\varepsilon_{00}$, and then access to reality in the real vacuum, whose permittivity is $\varepsilon_{0}=\varepsilon_{r} \varepsilon_{00}$. Condition from Equation (10) should be then better written in the variable

$$
\xi_{0}=\frac{4 \pi e}{\sqrt{2 \varepsilon_{00} c h}}
$$

whose $\xi_{\max }$ value sets the absolute vacuum permittivity at

$$
\varepsilon_{00}=\frac{1}{2 c h}\left(\frac{4 \pi e}{\xi_{\max }}\right)^{2}=8.753842149544 \times 10^{-12} \text { S.I. }
$$

and $\mathcal{\varepsilon}_{r}=1.0114630423466$.

It has to be noted that function $P$ attains its maximum for the variable $\xi$ solving the equation

$$
\tan \xi^{2}=2 \xi^{2},
$$

with a value $\xi_{\max }$ whose squared value is $(4 \pi)^{2}$ the fine structure constant value, increased by a factor due to the relative permeability factor $\mathcal{E}_{n}$ so that one may define a constant $\alpha_{0}=\varepsilon_{r} \alpha=0.00738100243079751$ in absolute vacuum, or $a_{0}^{-1}=135.482952528$.

\section{Evaluating the Vacuum Permittivity}

The consistency of the procedure finds its validation in obtaining else way the value of the permittivity of the absolute vacuum, or the susceptibility of the real vacuum

$$
\chi=\varepsilon_{r}-1=0.0114630423466 .
$$

In order to prospect such validation, let us look for the polarization produced in the vacuum, when a uniform electric field $E$ is present in a given volume of space. A number of virtual electric dipoles are constantly produced and annihilate in a way which depends upon the strength of the field $E$, each dipole lasting for a time $\Delta t$, inversely related to the energy needed to form it. The overall component of the dipoles along the electric field direction per unit volume 
stands as the electric polarization vector $\boldsymbol{P}$ of the vacuum, and its ratio to the electric field equals the product of the susceptibility constant $\chi$ of the vacuum times its permittivity

$$
\frac{\boldsymbol{P}}{\boldsymbol{E}}=\varepsilon_{0} \chi .
$$

In order to obtain the value of the permittivity, a procedure may be followed similar to the one previously followed for the charge maximum probability, by considering the product of the virtual dipole multiplied by its life-time $(p \Delta t)$. Indeed, the product of each charge times its displacement is to be taken into account in place of the dipole, so as to deal separately with the positive and the negative charge. The polarization electric field $\boldsymbol{E}_{\mathrm{P}}$ stands then as the conjugated variable, so that the contribution of the positive charge in the expression of the wave function may be written as

$$
\psi_{\mathrm{pol}}^{+} \propto \mathrm{e}^{i \frac{\boldsymbol{p} \cdot \boldsymbol{E}_{\mathrm{p}} \Delta t}{\hbar}}=\mathrm{e}^{i \frac{\boldsymbol{E}_{\mathrm{p}}}{\boldsymbol{E}}}
$$

where use has been done of the relation between time and energy. The wave function then writes as

$$
\psi_{\text {pol }}=\psi_{\text {pol }}^{+}+\psi_{\text {pol }}^{-} \propto \sin \left(\frac{\boldsymbol{E}_{\mathrm{p}}}{\boldsymbol{E}}\right)=\sin \chi .
$$

As for the case previously considered for the expected value of the charge, the expected value for the susceptibility $\chi$ will be the one that makes the function $\psi_{\text {pol }}$ to attain its maximum at

$$
\chi=\frac{\pi}{2}
$$

which is two orders of magnitude higher than the expected one. However, the electric polarization $\chi$ in the absolute vacuum is contributed by all the virtual particles, whose charge distribution follows the one previously reported in Equation (8), that gets its maximum for $\xi=\pi / 2$, where $\sin \xi=1$. Since the effective polarization in the real vacuum is contributed by those virtual charges alone that might interact with real charges, that is with electrons, the resulting maximum value $\pi / 2$ has to be multiplied by the value the charge function attains for the electronic charge value, that is by $\sin \alpha$, with $\alpha$ the fine structure constant. Expression $\sin \alpha$ is obtained from Equation (8), save for the factor $1 / 8 \pi$ in the phase expression. This is due to the electric field $E$ aligning the dipoles along its own direction, so that no integration over the solid angle $(4 \pi)$ has to be taken into account and that each charge contributes to just one half of the dipole intensity.

An additional effect has to be considered for the final evaluation of the susceptibility, which is the normalization factor of the probability function. The interaction, indeed, of the real charges with the electronic charges in the virtual field makes the wave function denominator to be reduced by the probability of the electron pair in the virtual field, that is by the amount $\sin ^{2} \alpha$. The interaction of a real charge, however, with one virtual pair finally releases an electronic 
charge of the same sign, whose probability

$$
\frac{1}{2} \frac{\sin ^{2} \alpha}{\left(\int_{-\infty}^{+\infty} \sin x^{2} d x\right)}=\frac{1}{2}\left(\frac{\sin \alpha}{\pi / 2}\right)^{2}
$$

has to be subtracted from the probability value $\sin ^{2} \alpha$ just reported above. Once, however, that this effect is taken into account, the function has to be newly normalized by subtraction of its probability term

$$
\left(\frac{\sin \alpha}{\pi / 2}\right)^{2} \frac{\pi}{2} \sin \alpha .
$$

The real electric susceptibility $\chi_{\text {real }}$ will be finally written as

$$
\chi_{\text {real }}=\frac{\pi}{2} \frac{\sin \alpha}{1-\sin ^{2} \alpha+\frac{1}{2}\left(\frac{\sin \alpha}{\pi / 2}\right)^{2}-\left(\frac{\sin \alpha}{\pi / 2}\right)^{2} \frac{\pi}{2} \sin \alpha},
$$

which is set equal to

$$
\chi_{\text {real }}=\frac{\alpha_{0}}{\alpha}-1,
$$

to give $\alpha=0.00729735256868569$, which matches the experimental value by a relative factor $8.41 \times 10^{-11}$. It should be noted that the $\alpha$ value of the fine structure constant is reported in the NIST data [3] with a standard uncertainty ranging from 0.0072973525682 to $\alpha=0.0072973525704$, between whose limit the value reported above is contained [10]. That sets a definite value to the relative $\mathcal{E}_{r}$ permittivity of vacuum, as it results from Equation (14).

\section{Conclusions}

In conclusion, two steps are produced for analytically deriving the value of the fine structure constant. Firstly, a wave function is constructed describing the generation of two electrical charges in the so-called absolute vacuum that lately do separate as free charges in the real vacuum. In this way, the obtained value for the electrical permittivity results to be slightly higher than the experimental one, due to the effect of virtual charges acting in the separation process. A second step is then produced, in order to evaluate the contribution of this effect on the value of the permittivity through the introduction of a wave function for the polarization vector that leads to the value of the finite structure constant, equal to the one available in the NIST data.

The procedure relays on the search of the wave functions describing the underlining physical processes and on their relative maxima determination, and its validity really stands upon the optimum match between the derived numerical value of the fine structure constant and its experimental datum. Slight variations introduced into the procedure, particularly into the normalization function of the susceptibility, do greatly alter the deduced value in a way which seems to be impracticable for valuable results. 


\section{Acknowledgements}

The author wishes to acknowledge Massimo Germano for constant valuable discussions and suggestions and Andrea Bettucci for his constant interest in the progress of the research.

\section{Conflicts of Interest}

The author declares no conflicts of interest regarding the publication of this paper.

\section{References}

[1] Dirac, P.A.M. (1937) Nature, 139, 323. https://doi.org/10.1038/139323a0

[2] Eddington, A. (1942) Proceedings of the Royal Physical Society, 54, 491-504. https://doi.org/10.1088/0959-5309/54/6/303

[3] https://physics.nist.gov/cgi-bin/cuu/Value?alph

[4] Sommerfeld, A. (1916) Annalen der Physik, 51, 1-94, 125-167. https://doi.org/10.1002/andp.19163561802

[5] Kragh, H. (2003) Archive for History of Exact Sciences, 57, 395-431. https://doi.org/10.1007/s00407-002-0065-7

[6] Sherbon, M. (2019) Fine-Structure Constant from Sommerfeld to Feynman. https://doi.org/10.31219/osf.io/hk8p5

[7] Jaeger, G. (2019) Entropy, 21, 141. https://doi.org/10.3390/e21020141

[8] Bohm, D. (1952) Physical Review, 85, 166-179. https://doi.org/10.1103/PhysRev.85.166

[9] Abbott, B.P., LIGO and Virgo Collaborations (2017) Physical Review Letters, 119, 161101-161117.

[10] Feynman, R.P. (1985) QED: The Strange Theory of Light and Matter. Princeton University Press, Princeton, 129-130. 